\title{
One-dimensional photonic crystal: The Kronig-Penney model
}

\author{
Subodha Mishra and S. Satpathy \\ Department of Physics \& Astronomy, University of Missouri, Columbia, Missouri 65211, USA
}

(Received 31 October 2002; published 30 July 2003)

\begin{abstract}
We formulate the photonic band-structure problem for a one-dimensional photonic crystal in terms of the reflection and transmission coefficients, obtaining a transcendental photonic band equation. The reflection and the transmission coefficients may be evaluated by using the standard transfer-matrix method. The structure of the equation reveals the existence of gaps, analogous to the Kronig-Penney model in the electronic bandstructure problem. As an example, the photonic band equation is solved for the simple case of the "KronigPenney" dielectric structure, consisting of alternating slabs of refractive indices $n_{1}$ and $n_{2}$.
\end{abstract}

DOI: 10.1103/PhysRevB.68.045121

PACS number(s): 42.70.Qs

\section{INTRODUCTION}

The idea of photonic crystals was demonstrated by the early experiments of Yablonovitch and Gmitter $^{1}$ and the flurry of theoretical works that followed. ${ }^{2}$ These crystals are microstructured materials in which the dielectric constant is periodically modulated with the result that the multiple interference of light scattered from different unit cells in the crystal produces a photonic band structure, where gaps may occur. The existence of a photonic gap leads to a number of physical properties, of both fundamental interest and for potential device applications.

In this paper, we derive a transcendental equation for the photonic band structure, akin to the solution of the KronigPenney model arising in the electronic band-structure problem. The equation, written in terms of the reflection and transmission coefficients of the unit cell of the crystal, allows us to derive several general properties of the photonic band structure. The method presented here is an alternative procedure to the plane-wave-expansion method, commonly used to solve the photonic band-structure problem. Some general relations between the reflection and transmission coefficients for the electromagnetic waves are also presented in the Appendix.

\section{MAXWELL'S EQUATIONS}

The Maxwell's equations for an electromagnetic (em) wave propagating through a dielectric medium with no free charge or current are given by

$$
\begin{gathered}
\vec{\nabla} \times \vec{E}=i \omega \vec{B}, \\
\vec{\nabla} \times \vec{H}+i \omega \vec{D}=0, \\
\vec{\nabla} \cdot \vec{D}=0, \\
\vec{\nabla} \cdot \vec{B}=0,
\end{gathered}
$$

where the time dependence of the em field has been taken to be $\vec{E}(\vec{r}, t)=\vec{E}(\vec{r}) e^{-i \omega t}$ and $\vec{B}(\vec{r}, t)=\vec{B}(\vec{r}) e^{-i \omega t}$. Furthermore, we also have

$$
\vec{D}(\vec{r})=\epsilon(\vec{r}) E(\vec{r})
$$

In the following, the dielectric constant $\epsilon(\vec{r})$ is translationally invariant along the $y$ and $z$ directions, varying only along the $x$ direction, in a periodic manner. (See Fig. 1.) Meanwhile, the magnetic permeability is taken to be uniform throughout this paper, being equal to its vacuum value $\mu(r)=\mu_{0}$, which is an excellent approximation for practical systems of interest.

Combining now the Maxwell's equations, we get the second-order differential equation for the displacement field $\vec{D}$ :

$$
\vec{\nabla} \times \vec{\nabla} \times[\vec{D}(r) / \epsilon(\vec{r})]=\omega^{2} \mu_{0} \vec{D} .
$$

The dielectric can be broken down into segments of constant $\epsilon$ as indicated in Fig. 1, so that the electric field $\vec{E}$ satisfies the equation in each segment,

$$
\nabla^{2} \vec{E}=-\frac{\omega^{2} n^{2}}{c^{2}} \vec{E},
$$

where $c$ is the vacuum speed of light and $n$ is the refractive index for the segment.

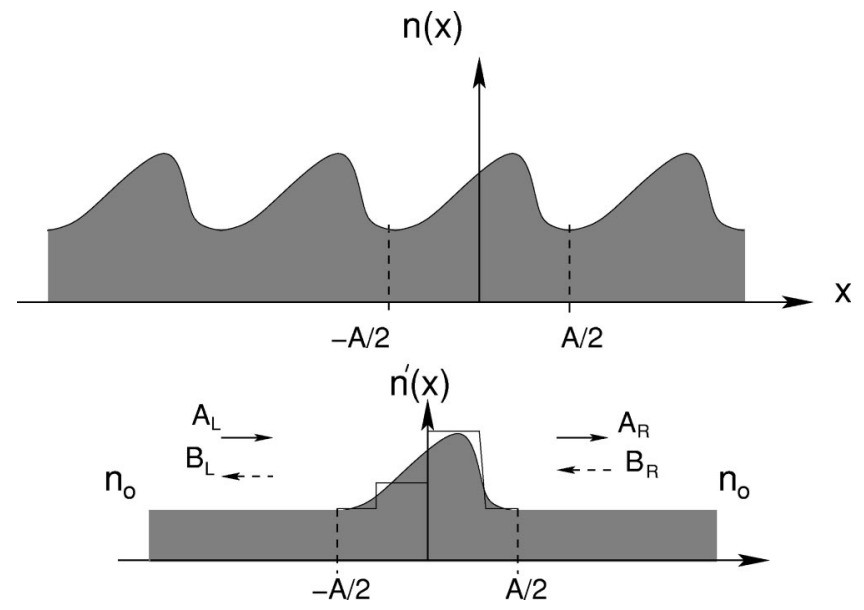

FIG. 1. Variation of the refractive index $n(x)$ for the onedimensional photonic crystal and its segmentation into regions of constant $n$ (shown in the lower part). The lower part of the figure shows the refractive index of one unit cell, attached to the asymptotic regions of the constant refractive index $n_{0}$ outside the cell. The quantities $n(x)$ and $n^{\prime}(x)$ are identical inside the unit cell. 


\section{PHOTONIC BAND EQUATION}

\section{A. Bloch periodicity}

In view of the fact that we have translational symmetry along directions parallel to the slab and Bloch periodicity along the direction perpendicular to the slab, the electric field may be written as

$$
\begin{gathered}
\vec{E}(\vec{r})=e^{i \vec{k}\|\cdot \vec{r}\|} \times \vec{F}(x), \\
\vec{F}(x)=\sum_{m} e^{i k_{B} m A} \vec{\xi}(x-m A),
\end{gathered}
$$

where a Bloch form has been taken for $\vec{F}(x)$ by adding the functions $\vec{\xi}(x-m A)$, centered in the $m$ th cell, after multiplying them with the appropriate phase factors. Any function for $\vec{\xi}(x)$ is good enough as far as the Bloch symmetry is concerned, but it has to be constructed such that $\vec{F}(x)$ satisfies the Maxwell's equations everywhere. Here, $k_{B}$ is the "Bloch momentum" with $-\pi / A \leqslant k_{B} \leqslant \pi / A$ and $A$ is the lattice constant, $x$ is normal to the slabs, and $y$ and $z$ are parallel to the slabs. The solution is thus characterized by the symmetry labels $\left(\vec{k}_{\|}, k_{B}\right)$ and given a specific value of these, our goal is to find $\omega$. This would give us all possible solutions for the em fields (photonic band structure).

Putting the expression for the electric field (5) into the wave Eq. (4), one finds that the equation for the field $\vec{F}(x)$ is

$$
\frac{d^{2} \vec{F}}{d x^{2}}=-k_{x, i}^{2} \vec{F}(x)
$$

where $k_{x, i}^{2}=\omega^{2} n_{i}^{2} / c^{2}-k_{\|}^{2}$. Thus $\vec{k}_{\|}$is fixed, while $k_{x, i}$ varies from segment to segment, according to the value $n_{i}$ corresponding to the $i$ th segment.

\section{B. Tail cancellation}

We first obtain the solution of the Maxwell's equation by the method of "tail cancellation," which although a bit tedious in one dimension as compared to the method using the boundary condition matching, is quite powerful for higherdimensional systems. The derivation below illustrates the method and can be generalized to two- and threedimensional photonic crystals. Similar generalization involving the "tail cancellation" is used in the formulation of the muffin-tin orbitals method in the electronic band-structure theory. ${ }^{3}$

The auxiliary functions $\vec{\xi}(x)$ are to be determined such that the Bloch sum Eq. (6) satisfies Eq. (7). Let us choose it in the following way: In the central cell (defined as $-A / 2$ $\leqslant x \leqslant A / 2), \quad \vec{\xi}(x)$ satisfies Eq. (7), so that

$$
\frac{d^{2} \vec{\xi}(x)}{d x^{2}}=-k_{x, i}^{2} \vec{\xi}(x)
$$

while outside the central cell, the "tail" of $\vec{\xi}(x)$ is such that the function is well behaved (continuous and differentiable) at the cell boundary. This will ensure that the Bloch sum of such functions is well behaved whenever $n(x)$ is smoothly varying.

The tail may be chosen such that it satisfies the Maxwell's equation, Eq. (8), for some $n(x)$ in the tail region, but it is not necessary that it does so (since the tails will cancel out). It is convenient to choose the tails of $\vec{\xi}(x)$ to satisfy the Maxwell's equation with a constant $n_{0} \equiv n(x=A / 2)$, i.e., the same refractive index as its value at the boundaries of the central cell. That way we can compute the transmission and reflection coefficients easily, which are needed later. The refractive index $n^{\prime}(x)$ that $\vec{\xi}(x)$ sees in the entire space is indicated in the lower part of Fig. 1, while the upper part of the same figure indicates the refractive index $n(x)$ seen by the function $\vec{F}(x)$. The refractive indices $n(x)$ and $n^{\prime}(x)$ are the same in the central cell, differing from each other only outside the cell.

To find a solution, $\omega\left(\overrightarrow{k_{\|}}, k_{B}\right)$, let us try a specific guess solution $\omega$ for the given values of $\overrightarrow{k_{\|}}$and $k_{B}$. First we construct the auxiliary function $\vec{\xi}(x)$ for the central cell with these $\overrightarrow{k_{\|}}$and $\omega$ from the differential equation, Eq. (8), in terms of which the electric field $\vec{F}(x)$ for the entire periodic structure will be given by the Bloch sum Eq. (6). This will be a solution for the entire dielectric structure, provided that the Bloch sum of the tails of the auxiliary functions cancel everywhere. In that case, the Bloch sum will satisfy the Maxwell's equation everywhere, because only the "heads" of the auxiliary functions survive, which by explicit construction satisfy the Maxwell's equation within the central cell. The condition that the tails will cancel in the central cell (and therefore in all other cells) is that

$$
\sum_{m \neq 0} e^{i k_{B} m A} \vec{\xi}(x-m A)=0
$$

for all values of $x$ in the central cell. The idea of the "tail cancellation" is in fact well known from the Korringa-KohnRostoker Green's function and the muffin-tin orbital formulations of the electronic band-structure theory. ${ }^{3}$

We now turn to the construction of $\vec{\xi}(x)$, the explicit form of which is needed just for the tail, in order to apply the tail cancellation condition. Now, since the geometry of our problem is such that TE and TM modes do not mix, ${ }^{4}$ and further, since in regions with constant $\epsilon(x), \vec{\nabla} \cdot \vec{E}=0$ implies that the $\vec{E}$ fields are transverse to the direction of propagation, we can work with the two modes separately, with the mode index $\lambda=1$ and 2. For each of the modes, we have two solutions, right and left propagating, respectively, which we call $\vec{\xi}_{1}(x)$ and $\vec{\xi}_{2}(x)$. Written in terms of the reflection and the transmission coefficients, $r_{\lambda}$ and $t_{\lambda}$, the four independent solutions for the auxiliary functions $\vec{\xi}(x)$ satisfying Eq. (8) are then

$$
\vec{\xi}_{1}^{\lambda}(x)= \begin{cases}\left(e^{i k_{x} x}+r_{\lambda} e^{-i k_{x} x}\right) \hat{e}_{\lambda}, & x \leqslant-A / 2, \\ t_{\lambda} e^{i k_{x} x} \hat{e}_{\lambda}, & x \geqslant A / 2,\end{cases}
$$




$$
\vec{\xi}_{2}^{\lambda}(x)= \begin{cases}t_{\lambda} e^{-i k_{x} x} \hat{e}_{\lambda}, & x \leqslant-A / 2, \\ \left(e^{-i k_{x} x}+r_{\lambda}^{\prime} e^{i k_{x} x}\right) \hat{e}_{\lambda}, & x \geqslant A / 2,\end{cases}
$$

where

$$
k_{x}^{2} \equiv \omega^{2} / c^{2} \times n_{0}^{2}-k_{\|}^{2}
$$

has the fixed value corresponding to the tail region, and the unit vector $\hat{e}_{\lambda}$ indicates the direction of the electric field corresponding to the polarization $\lambda$. Note that since the values of $\epsilon$ and $\mu$ are same at the left and the right cell boundaries for a periodic crystal, the transmission coefficients are the same, while the reflection coefficients $r$ and $r^{\prime}$ differ by a phase factor [see Eq. (A21)].

Note also that the above expressions are only for the tails, since, at this point, we don't really care how the auxiliary electric fields look inside the central cell. The electric field may be obtained everywhere by integration of the Maxwell's equations, Eq. (8), once the eigenfrequency $\omega$ has been determined.

Since the TE and the TM modes don't mix, the most general solution for $\vec{\xi}(x)$ is written as a linear combination of the two independent solutions for each mode,

$$
\vec{\xi}(x)=A_{\lambda} \vec{\xi}_{1}^{\lambda}(x)+B_{\lambda} \vec{\xi}_{2}^{\lambda}(x),
$$

where, again, $\lambda=1$ and 2 , corresponding to the TE and TM modes, respectively. Substituting the expression for $\vec{\xi}(x)$ in the tail cancellation condition Eq. (9) and equating the coefficients of $e^{ \pm i k_{x} x}$ in the resulting expression to be zero, we obtain the conditions that the coefficients $A_{\lambda}$ and $B_{\lambda}$ must satisfy:

$$
\begin{aligned}
& A_{\lambda}\left[S_{-}+t_{\lambda} S_{-}^{*}\right]+B_{\lambda} r_{\lambda}^{\prime} S_{-}^{*}=0, \\
& A_{\lambda} r_{\lambda} S_{+}+B_{\lambda}\left[t_{\lambda} S_{+}+S_{+}^{*}\right]=0,
\end{aligned}
$$

where $S_{ \pm} \equiv S\left(k_{B} \pm k_{x}\right)$ with $S(k) \equiv \sum_{m=1}^{\infty} \exp (i A m k)$.

The sum $S(k)$ is over a series of oscillating terms. The oscillation can be traced to the fact that the plane-wave-like tails in Eq. (10) continue undamped to infinity. If we keep a finite number of terms $N$ in the summation, then the second term in the numerator of the result $S(k)=\left[e^{i k A}\right.$ $\left.-e^{i k(N+1) A}\right] /\left(1-e^{i k A}\right)$ oscillates rapidly between -1 and +1 as $N \rightarrow \infty$ with the average value zero. It turns out that taking this average value yields the correct answer for the problem at hand. A more careful way of evaluating the sum is to take the limit

$$
S(k)=\lim _{N \rightarrow \infty, \mu \rightarrow 0} \sum_{n=1}^{N} e^{(i k-\mu) n A}=\frac{e^{i k A}}{1-e^{i k A}},
$$

where the limit has been taken in such a way that $\mu A \ll 1$ and $\mu N A \gg 1$. Physically this corresponds to a small damping term $e^{-\mu|x|}$ in the auxiliary functions, Eq. (10), such that the amplitudes of the plane-wave tails damp out at infinity but do not change appreciably over the length of a unit cell. The same situation arises in the solution of the Kronig-Penney model by the tail cancellation method in the electronic problem as well. ${ }^{5}$

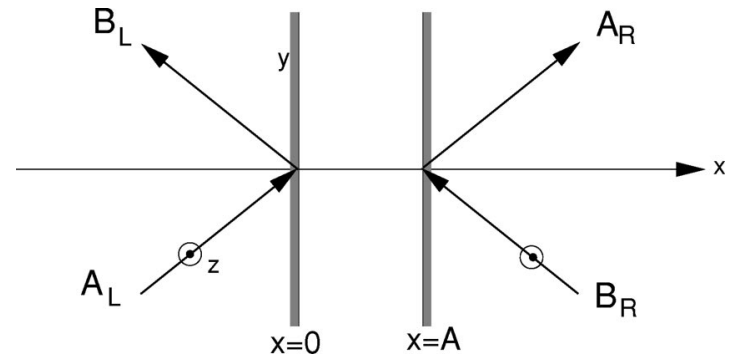

FIG. 2. Sketch of the unit cell for obtaining the photonic band equation using the boundary condition matching for the TE mode. Electric-field coefficients $(A$ and $B$ ) on the left and right sides of the cell are valid in the small shaded region, where the dielectric constants may be taken to be unchanged.

Eliminating now the unknowns $A_{\lambda}$ and $B_{\lambda}$ from Eq. (13) and after some algebra, we get the following transcendental equation for $\omega$ :

$$
\left(\frac{t_{\lambda}^{2}-r_{\lambda} r_{\lambda}^{\prime}}{2 t_{\lambda}}\right) e^{i k_{x} A}+\frac{e^{-i k_{x} A}}{2 t_{\lambda}}=\cos \left(k_{B} A\right) .
$$

Using Eq. (A21) of the Appendix, we write the left and the right transport coefficients in terms of their magnitudes and phases:

$$
\begin{gathered}
t_{\lambda}=t_{\lambda}^{\prime}=\left|t_{\lambda}\right| e^{i \eta_{\lambda}}, \\
r_{\lambda}=\left|r_{\lambda}\right| e^{i \delta_{\lambda}}, \\
r_{\lambda}^{\prime}=-\left|r_{\lambda}\right| e^{i\left(2 \eta_{\lambda}-\delta_{\lambda}\right)} .
\end{gathered}
$$

Putting this in Eq. (15), we get

$$
\frac{\cos \left(k_{x} A+\eta_{\lambda}\right)}{\left|t_{\lambda}\right|}=\cos \left(k_{B} A\right) .
$$

This is the central equation of the paper, the solution of which gives the photonic band structure. It is very similar to the equation appearing in the Kronig-Penney model for the electronic case $;^{5,6}$ however, unlike the electronic case, we now have two modes corresponding to the two polarizations of light, $\lambda=1,2$. The band structure is expressed here in terms of the complex transmission coefficient $t$ for the unitcell dielectric structure embedded in a uniform dielectric of refractive index $n_{0}$ on either side. As we shall see later explicitly, the dependence of $n_{0}$ drops out of the photonic band equation as it must; however, it is needed to define the tranmission coefficient.

\section{Photonic band equation from boundary matching}

The photonic band equation can be obtained more simply by matching the boundary condition. Consider first the TE mode. This is fine since for a stratified medium the TE and TM modes don't mix. ${ }^{4}$ Referring to Fig. 2, the electric fields in the immediate neighborhood on the left and right sides of the unit cell may be expressed as

$$
\vec{E}_{L}=e^{i \vec{k} \| \cdot \vec{r}} \| \times\left(A_{L} e^{i k_{x} x}+B_{L} e^{-i k_{x} x}\right) \hat{z}
$$




$$
\begin{aligned}
\vec{E}_{R}= & e^{i \vec{k}_{\|} \cdot \vec{r}_{\|}} \times\left[\left(s_{11} A_{L}+s_{12} B_{L}\right) e^{i k_{x} x}\right. \\
& \left.+\left(s_{21} A_{L}+s_{22} B_{L}\right) e^{-i k_{x} x}\right] \hat{z},
\end{aligned}
$$

where the right coefficients $A_{R}$ and $B_{R}$ have been written in terms of $A_{L}$ and $B_{L}$ using the scattering matrix (Appendix $\mathrm{A}$ ), and the wave vector $k_{x}$ is related to the frequency $\omega$ via Eq. (11). In this formulation, the refractive index $n_{0}$ entering in that equation is the refractive index at the cell boundary as indicated in the shaded area in Fig. 2.

Now, the continuity conditions at the boundary, viz., that $E_{\|}$and $H_{\|}$are continuous, together with the Maxwell's equation relating $\vec{\nabla} \times \vec{E}$ to $\vec{B}$ and the Bloch's theorem, lead to the two equations:

$$
\begin{gathered}
\vec{E}_{R}(A)=e^{i k_{B} A} \vec{E}_{L}(0), \\
\left(d \vec{E}_{R} / d x\right)_{A}=e^{i k_{B} A}\left(d \vec{E}_{L} / d x\right)_{0} .
\end{gathered}
$$

Substituting these into Eq. (18), we get the eigenvalue equation

$$
\left(\begin{array}{cc}
s_{11} e^{i k_{x} A} & s_{12} e^{i k_{x} A} \\
s_{21} e^{-i k_{x} A} & s_{22} e^{-i k_{x} A}
\end{array}\right)\left(\begin{array}{c}
A_{L} \\
B_{L}
\end{array}\right)=e^{i k_{B} A}\left(\begin{array}{c}
A_{L} \\
B_{L}
\end{array}\right) .
$$

The Bloch factor $e^{i k_{B} A}$ is therefore an eigenvalue of the $2 \times 2$ matrix, ${ }^{7,8}$ the structure of which is such that (see the Appendix) if $\lambda$ is an eigenvalue then so is $\lambda^{*}$. And, since the trace is unchanged by diagonalization, one gets

$$
s_{11} e^{i k_{x} A}+s_{22} e^{-i k_{x} A}=2 \cos \left(k_{B} A\right) .
$$

Expressing now the elements of the $S$ matrix appearing in the above equation in terms of the transport coefficients [Eqs. (A9) and (A17)], viz.,

$$
s_{11}=s_{22}^{*}=1 / t^{*},
$$

we obtain the same photonic band equation as Eq. (17). For the TM mode, the same equation may be derived by working with the $\vec{B}$ field and following similar arguments.

\section{SOLUTION OF THE PHOTONIC BAND EQUATION}

\section{A. Structure of the equation}

The photonic band-structure Eq. (17) is of the form

$$
f\left(\vec{k}_{\|}, k_{B}, \omega\right)=0 .
$$

Another auxiliary variable which is quite physical is the angle of incidence defined as

$$
\sin \theta=\frac{\left|\vec{k}_{\|}\right|}{K}
$$

where $K \equiv \omega n / c$. Since the refractive index varies along $x$, so do the quantities $K$ and $\theta$. With this, the photonic band equation has the form

$$
f\left(\theta_{\text {inc }}, k_{B}, \omega\right)=0,
$$
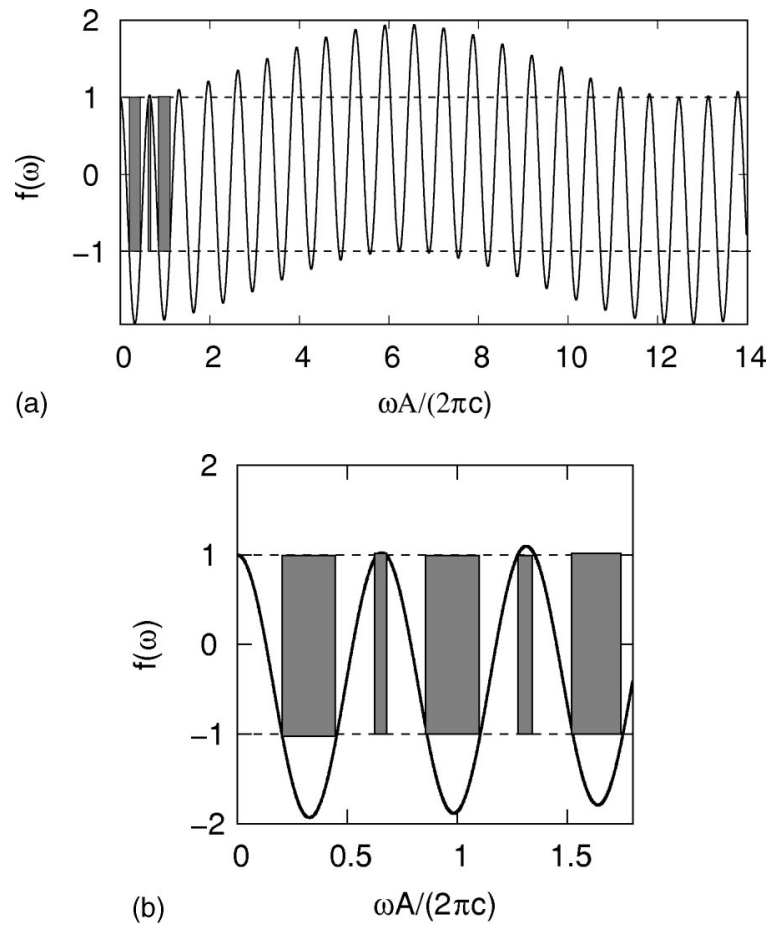

FIG. 3. Plot of the function $f(\omega)=\cos \bar{\eta}_{\lambda} /\left|\bar{t}_{\lambda}\right|$, for a slab dielectric structure for the case of normal incidence $\vec{k}_{\|}=0$. As indicated by the photonic band equation, Eq. (17), those values of $\omega$ are allowed for which the function lies between \pm 1 . For other frequencies, there is no Bloch solutions so that forbidden gaps in the frequency spectrum are formed, the first few of which have been shaded in the figure. Lower part of the figure shows an enlargement in the lower-frequency region. Dielectric parameters are $\epsilon=13$ for $|x| \leqslant 0.2 A$, and 1 for $0.2 A \leqslant|x| \leqslant A$ in the unit cell of the crystal.

$\theta_{\text {inc }}$ being the angle of incidence at a reference point (say, the edge of the unit cell, $x= \pm A / 2$ with refractive index $n_{0}$ ). Since $\vec{k}_{\|}$is a constant throughout the structure, so is $K \sin \theta$ [Eq. (24)], from which it follows that $n \sin \theta$ is also the same throughout the system. This is nothing but Snell's law, which is seen here to be a consequence of the translational symmetry.

The problem then boils down to solving for $\omega_{\lambda}$, given the symmetry labels ("Bloch momentum") $\vec{k}_{\|}$and $k_{B}$, or, equivalently, given $\theta_{i n c}$ and $k_{B}$. The solution of the transcendental Eq. (17) is illustrated in Fig. 3, which shows the occurrence of gaps in the photonic spectrum.

\section{B. Computation of the transmission coefficient}

In order to solve the photonic band equation, Eq. (17), we need to compute the transmission coefficient $t_{\lambda}$. This is most simply computed by using the transfer-matrix method as outlined below, a method that is quite familiar in the treatment of light transmission in layered media. ${ }^{7,9-11}$

We first divide the cell into a large number $N$ of segments along $x$ such that the refractive index in each segment may be considered to be a constant. For each of the two modes (TE or TM), the em fields at the left and right end of the ith segment are related via the $2 \times 2$ transfer matrix $\mathcal{M}_{i}$, 


$$
\left(\begin{array}{c}
E_{a} \\
B_{a}
\end{array}\right)=\mathcal{M}_{i}\left(\begin{array}{c}
E_{b} \\
B_{b}
\end{array}\right)
$$

with the numerical value,

$$
\begin{aligned}
& \mathcal{M}_{i}=\left(\begin{array}{cc}
\cos \delta_{i} & i \sin \delta_{i} / \gamma_{i} \\
i \gamma_{i} \sin \delta_{i} & \cos \delta_{i}
\end{array}\right), \\
& \delta_{i}=-d_{i} \bar{k}_{i} \quad(\text { TE } \text { or } \mathrm{TM}), \\
& \gamma_{i}^{\lambda}= \begin{cases}\bar{k}_{i} / \omega & (\mathrm{TE}), \\
\left(n_{i} / c\right)^{2} /\left(\bar{k}_{i} / \omega\right) & (\mathrm{TM}),\end{cases}
\end{aligned}
$$

with

$$
\bar{k}_{i}=\left\{\left(\omega n_{i} / c\right)^{2}-k_{\|}^{2}\right\}^{1 / 2},
$$

where $n_{i}$ is the refractive index of the $i$ th segment, $d_{i}$ is its thickness $\left(\sum_{i=1}^{N} d_{i}=A\right)$, and $c$ is the vacuum speed of light.

The overall transfer matrix corresponding to the entire unit cell, treated as a multisegment stack consisting of $N$ segments, is the product of the individual transfer matrices:

$$
\mathcal{M} \equiv\left(\begin{array}{ll}
m_{11} & m_{12} \\
m_{21} & m_{22}
\end{array}\right)=\lim _{N \rightarrow \infty} \prod_{i=1}^{N} \mathcal{M}_{i} .
$$

The transmission and the reflection coefficients for the entire multilayer stack may be written in terms of the following two quantities:

$$
\begin{gathered}
\bar{t}=2 \Gamma_{0} /\left(\Gamma_{0} m_{11}+\Gamma_{0}^{2} m_{12}+m_{21}+\Gamma_{0} m_{22}\right), \\
\bar{r}=\frac{\left(\Gamma_{0} m_{11}+\Gamma_{0}^{2} m_{12}-m_{21}-\Gamma_{0} m_{22}\right)}{\left(\Gamma_{0} m_{11}+\Gamma_{0}^{2} m_{12}+m_{21}+\Gamma_{0} m_{22}\right)},
\end{gathered}
$$

where

$$
\Gamma_{0}=1 / \omega \times\left\{\left(\omega n_{0} / c\right)^{2}-k_{\|}^{2}\right\}^{1 / 2}
$$

corresponds to the tail region, $n_{0}$ being the refractive index at the two edges of the unit cell, $x= \pm A / 2$. The quantities $\bar{t}$ and $\bar{r}$ are in fact the transmission and reflection coefficients, respectively, if we define them according to their values at the left and the right boundaries (see, e.g., Pedrotti and Pedrotti ${ }^{9}$ ). We have, in contrast, defined $t$ and $r$ according to their asymptotic forms, Eq. (10).

Note that the transfer matrices depend on the parameters of the slab only, without reference to the dielectric in which it is embedded, while the reflection and the transmission coefficients depend on the refractive index of the embedding medium $\left(n_{0}\right)$, the dependence entering via the quantity $\Gamma_{0}$ in Eq. (29).

The barred and the unbarred coefficients $(t, r, \bar{r}$, and $\bar{t})$ are related quite simply. Since for the construction of the auxiliary functions $\vec{\xi}(x)$ in Eq. (10), we have taken the dielectric constant to be fixed $[\epsilon(x)=\epsilon(|A / 2|)$ for $x \geqslant|A / 2|]$, the asymptotic forms extend right up to the unit-cell boundary. Thus the em fields at the cell boundaries can be written in terms of $r$ and $t$, and a connection made with the barred quantities. Following these lines, one finds that the barred and the unbarred coefficients differ only by a phase factor:

$$
t=\bar{t} \times e^{-i k_{x} A}, \quad r=\bar{r} \times e^{i k_{x}\left(2 x_{0}\right)},
$$

where the unit cell extends between $x=x_{0}$ and $x=x_{0}+A$. In terms of the barred transmission coefficients for the two polarizations $\lambda$,

$$
\bar{t}_{\lambda}=\left|\bar{t}_{\lambda}\right| \times e^{i \bar{\eta}_{\lambda}},
$$

the photonic gap Eq. (17) is then written as

$$
\frac{\cos \bar{\eta}_{\lambda}}{\left|\bar{t}_{\lambda}\right|}=\cos \left(k_{B} A\right) \text {. }
$$

While the photonic band equation [Eq. (33)] is quite physical, being analogous to the corresponding equation in the electronic case, and it provides the connection between the transmission coefficient and the photonic bands, it may be simplified somewhat for practical calculations by writing it in terms of the transfer matrix $\mathcal{M}$. We first note that the diagonal elements of $\mathcal{M}$ are real, while the off-diagonal elements are purely imaginary, a form that originates from the matrix form for the individual segment $\mathcal{M}_{i}$ and is retained as we multiply such matrices to obtain the final $\mathcal{M}$. Therefore, in the expression for $\mathcal{M}$ [Eq. (28)], $m_{11}$ and $m_{22}$ are real, while $m_{12}$ and $m_{21}$ are purely imaginary. Using this fact in evaluating the left part of the photonic band equation [Eq. (33)] by plugging in the transmission coefficient [Eq. (29)], we find that the photonic band equation may alternatively be written as

$$
1 / 2 \times \operatorname{Tr} \mathcal{M}=\cos \left(k_{B} A\right) .
$$

The equation may also be obtained directly from the boundary condition matching. ${ }^{10}$ The three equivalent equations for the photonic band structure [Eqs. (17), (33), and (34)] are the central equations of the paper.

It is clear from Eq. (34) that the band structure is independent of the constant $n_{0}$, since it never appears in the expression for $\mathcal{M}$. Secondly, the band structure is independent of the choice of the unit cell. This is simply because the trace of a product of matrices is unchanged under cyclic permutation of the matrices, $\operatorname{Tr}(A B C)=\operatorname{Tr}(C A B)$, and in our formulation, choosing a different unit cell amounts to multiplication of the same set of transfer matrices, without altering the sequence, e.g., $\mathcal{M}_{1} \times \mathcal{M}_{2} \times \mathcal{M}_{3} \times \mathcal{M}_{4}$ vs $\mathcal{M}_{2}$ $\times \mathcal{M}_{3} \times \mathcal{M}_{4} \times \mathcal{M}_{1}$, if the unit cell is divided into four segments.

\section{Weak scatterers}

We consider now the limiting case of a weak scatterer and normal incidence $\left(\overrightarrow{k_{\|}}=0\right)$. For a weak scatterer defined as $|t| \approx 1,|r| \approx 0$, and $\eta \approx 0$, the transcendental Eq. (17) shows that the band gaps are centered around the wave vector $k_{0} A=m \pi, m$ being an integer. Band states begin with nearby $k_{x}$ values of $k_{ \pm}=k_{0} \pm \alpha$, so that 


$$
\left|\cos \left(k_{0} A \pm \alpha A\right)\right|=|t|,
$$

since at these values the right-hand side of Eq. (17) becomes plus or minus one. Solving this, with the assumption that $r$ does not vary strongly in the gap region, one finds the value for the gap to be

$$
\Delta \omega=c\left(k_{+}-k_{-}\right) / n_{0}=\frac{2 c}{n_{0} A} \times|r|,
$$

where $r$ is the reflection coefficient for some average frequency $\omega$ in the gap region. Thus the band gap scales linearly with the magnitude of the reflection coefficient in the limit that it is small.

In the case of the strong scatterers, the transmission coefficient as a function of the frequency $\omega$ is small much of the time except for sharp resonances. Photonic bands will be narrow, forming in the region in which the resonances occur, i.e., $|t(\omega)| \approx 1$, with the bandwidth controlled by the sharp variation of the complex $t(\omega)$ with $\omega$. Apart from these bands, only in rare cases will the cosine term in the numerator in Eq. (17) conspire with a small $|t|$ in the denominator to produce a ratio of magnitude less than unity to provide additional bands away from the resonances.

\section{Numerical results}

We now turn to the calculation of the photonic band structure for a specific example. We consider a Kronig-Penney slab dielectric, consisting of alternate slabs of dielectric constants $n_{2}$ and $n_{1}$, of thicknesses $a$ and $b$, respectively, with the unit-cell size $A=a+b$. The ratio $\cos \bar{\eta}_{\lambda} /\left|\bar{t}_{\lambda}\right|$ appearing in the photonic band equation [Eq. (33)] is easily obtained by constructing and multiplying two $\mathcal{M}$ matrices following Sec. IV B.

There are just two $\mathcal{M}$ matrices involved corresponding to the two slabs, viz.,

$$
\mathcal{M}_{1}=\left(\begin{array}{cc}
\cos \delta_{1} & i \sin \delta_{1} / \gamma_{1} \\
i \gamma_{1} \sin \delta_{1} & \cos \delta_{1}
\end{array}\right),
$$

and

$$
\mathcal{M}_{2}=\left(\begin{array}{cc}
\cos \delta_{2} & i \sin \delta_{2} / \gamma_{2} \\
i \gamma_{2} \sin \delta_{2} & \cos \delta_{2}
\end{array}\right),
$$

which are obtained from Eq. (27). Multiplying the two matrices and taking the trace of the resulting matrix $\mathcal{M}=\mathcal{M}_{1}$ $\times \mathcal{M}_{2}$, we get the photonic band equation

$$
-\frac{1}{2} \times\left(\frac{\gamma_{1}}{\gamma_{2}}+\frac{\gamma_{2}}{\gamma_{1}}\right) \sin \delta_{1} \sin \delta_{2}+\cos \delta_{1} \cos \delta_{2}=\cos \left(k_{B} A\right) .
$$

The gammas and the deltas for the two slabs are defined in Eq. (27) and note that they are different for the TE and the TM modes, leading to two different equations for the two

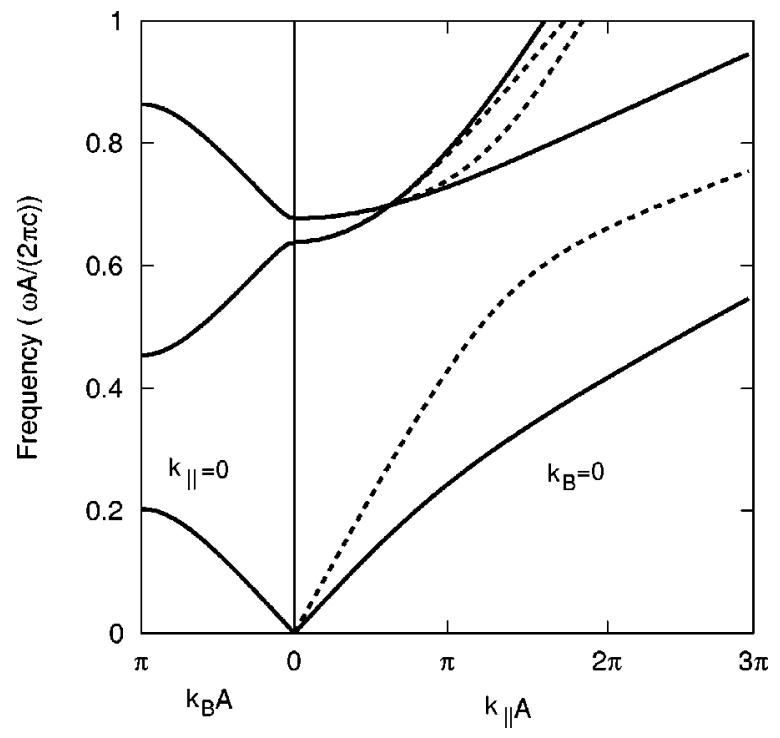

FIG. 4. Photonic band structure for the same slab dielectric structure considered in Fig. 3 for both normal $\left(\vec{k}_{\|}=0\right.$; left panel) and slanted $\left(\vec{k}_{\|} \neq 0\right.$; right panel) incidence. For the left panel, $\vec{k}_{\|}$ $=0$ and the Bloch momentum $k_{B}$ varies along the $x$ axis, while for the right panel, we have $k_{B}=0$ and varying $\vec{k}_{\|}$. For the slanted incidence, the TE and the TM modes have different frequencies and they are indicated by solid and dashed lines, respectively.

modes. However, for normal incidence $\overrightarrow{k_{\|}}=0$, the two equations become identical:

$$
-\frac{1}{2} \times\left(\frac{n_{1}}{n_{2}}+\frac{n_{2}}{n_{1}}\right) \sin \delta_{1} \sin \delta_{2}+\cos \delta_{1} \cos \delta_{2}=\cos \left(k_{B} A\right),
$$

so that for the case of normal incidence, TE and TM modes are degenerate.

The photonic band structure obtained by solving the transcendental Eq. (39) is plotted in Fig. 4. For normal incidence $\left(\vec{k}_{\|}=0\right)$, the TE and the TM modes are degenerate, as might be expected, while for slanted incidence $\left(\vec{k}_{\|} \neq 0\right)$, they are not. The results shown in Fig. 4 agree completely with earlier results for the same structure obtained by using the planewave-expansion method. ${ }^{12}$

\section{CONCLUSION}

In conclusion, we have obtained a transcendental equation for the photonic band structure for a one-dimensional photonic crystal in terms of the reflection and the transmission coefficients, analogous to the solution of the Kronig-Penney model in the electronic structure problem. The photonic band equation allows us to extract several general features of the photonic band structure and illustrates the origin of the band gaps. The case of the Kronig-Penney dielectric structure, with slabs of refractive index $n_{1}$ alternating with slabs of $n_{2}$, was solved as an illustration of the method. 

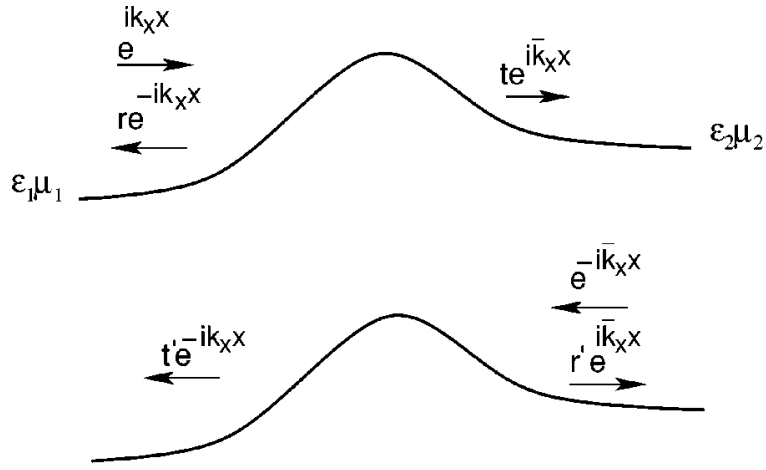

FIG. 5. Reflection and transmission coefficients for light incident from the left and the right. The coefficients are defined with reference to the plane-wave solutions in the asymptotic regions $x$ $\rightarrow \pm \infty$.

\section{ACKNOWLEDGMENTS}

We acknowledge support of this work by the U. S. Department of Energy (Grant Nos. DE-FG02-00ER45818).

\section{APPENDIX: REFLECTION AND TRANSMISSION COEFFICIENTS}

In this Appendix, we outline the relationship between the transmission and reflection coefficients for the electromagnetic waves incident on a dielectric. Consider the dielectric medium with the dielctric constant and the magnetic permeability varying along $x$ : $\epsilon(x)$ and $\mu(x)$ varying from one set of constants, $\epsilon_{1}, \mu_{1}$ as $x \rightarrow-\infty$, to another, $\epsilon_{2}, \mu_{2}$ as $x$ $\rightarrow \infty$, as in Fig. 5. In the absence of any free charge or current, the electric field obeys the Maxwell wave equation in the asymptotic region $(x \rightarrow \pm \infty)$ :

$$
\nabla^{2} \vec{E}=-\frac{\omega^{2} n^{2}}{c^{2}} \vec{E},
$$

where we have assumed a harmonic time dependence $\vec{E}(\vec{r}, t)=\vec{E}(\vec{r}) e^{-i \omega t}$, with $c$ the vacuum speed of light, and $n$ the refractive index, $n=\sqrt{\mu \epsilon} \times c$. Not just in the asymptotic region, but in all space one can in fact write down the above equation for $\vec{E}$, provided one divides the given $\epsilon(x), \mu(x)$ into piecewise segments of constants $\epsilon$ and $\mu$.

A similar wave equation for the magnetic field can be written, viz.,

$$
\nabla^{2} \vec{B}=-\frac{\omega^{2} n^{2}}{c^{2}} \vec{B}
$$

With our geometry, the TE and TM modes don't mix and it is convenient to work with the wave equation for $\vec{E}$ for the TE mode and the wave equation for $\vec{B}$ for the TM mode.

\section{TE mode: Asymptotic forms and the scattering matrix.}

Considering first the TE mode, the asymptotic form of the electric field is given, both for $x \rightarrow-\infty$ and for $x \rightarrow+\infty$, by a sum of two plane-wave components

$$
\begin{gathered}
\vec{E}=e^{i k_{y} y} \times\left(A_{L} e^{i k_{x} x}+B_{L} e^{-i k_{x} x}\right) \hat{z}, x \rightarrow-\infty, \\
\vec{E}=e^{i k_{y} y} \times\left(A_{R} e^{i \bar{k}_{x} x}+B_{R} e^{-i \bar{k}_{x} x}\right) \hat{z}, x \rightarrow \infty,
\end{gathered}
$$

where $\omega^{2} n_{1}^{2} / c^{2}=k_{x}^{2}+k_{y}^{2}, \omega^{2} n_{2}^{2} / c^{2}=\bar{k}_{x}^{2}+\bar{k}_{y}^{2}$, and we have chosen the $y$ direction along $\vec{k}_{\|}$(fixed throughout the structure owing to the translational symmetry along directions parallel to the slab) and the direction of polarization of $\vec{E}$ to be along $\hat{z}$.

Since these expressions are asymptotic forms of the same solution of a linear differential equation, there must be a linear relation between the coefficients, which defines the scattering matrix:

$$
\left(\begin{array}{l}
A_{R} \\
B_{R}
\end{array}\right)=\left(\begin{array}{ll}
s_{11} & s_{12} \\
s_{21} & s_{22}
\end{array}\right)\left(\begin{array}{l}
A_{L} \\
B_{L}
\end{array}\right) .
$$

It is clear from Eq. (A1) that if $\vec{E}$ is a solution, then so is $\vec{E}^{*}$. In addition, it also follows from Eq. (A1) that if $\vec{E}=e^{i \lambda y}$ $\times f(x) \hat{z}$ is a solution, then so is the function $\vec{E}=e^{-i \lambda y}$ $\times f(x) \hat{z}$. The asymptotic forms, obtained by taking the complex conjugate of Eq. (A3) and changing the sign of $k_{y}$,

$$
\begin{aligned}
& \vec{E}=e^{i k_{y} y} \times\left(A_{L}^{*} e^{-i k_{x} x}+B_{L}^{*} e^{i k_{x}^{x}}\right) \hat{z}, \\
& \vec{E}=e^{i k_{y} y} \times\left(A_{R}^{*} e^{-i \bar{k}_{x} x}+B_{R}^{*} e^{i \bar{k}_{x} x}\right) \hat{z},
\end{aligned}
$$

differ from Eq. (A3) only in the nomenclature of the constant coefficients. We therefore have

$$
\left(\begin{array}{l}
B_{R}^{*} \\
A_{R}^{*}
\end{array}\right)=\left(\begin{array}{ll}
s_{11} & s_{12} \\
s_{21} & s_{22}
\end{array}\right)\left(\begin{array}{l}
B_{L}^{*} \\
A_{L}^{*}
\end{array}\right) .
$$

Taking the complex conjugate and comparing with Eq. (A4), we obtain the relations between the $S$-matrix elements:

$$
\begin{aligned}
& s_{11}=s_{22}^{*}, \\
& s_{12}=s_{21}^{*} .
\end{aligned}
$$

\section{TE mode: Reflection and transmission coefficients.}

Meanwhile, let the reflection and transmission coefficients for a wave incident from the left be denoted by $r$ and $t$, while be let $r^{\prime}$ and $t^{\prime}$ denote the corresponding quantites for a wave incident from the right as indicated in Fig. 5. For the wave incident from the left, the asymptotic form of the electric field is given by

$$
\begin{gathered}
\vec{E}=e^{i k_{y} y} \times\left(e^{i k_{x} x}+r e^{-i k_{x} x}\right) \hat{z}, \\
\vec{E}=e^{i k_{y} y} \times\left(t e^{i \bar{k}_{x} x}\right) \hat{z} .
\end{gathered}
$$

This is a special case of the general asymptotic solution (A3) with $A_{L}=1, B_{L}=r, A_{R}=t$, and $B_{R}=0$. Putting these values into Eq. (A4), the $S$ matrix can be written as 


$$
S=\frac{1}{1-|r|^{2}}\left(\begin{array}{cc}
t & -t r^{*} \\
-t^{*} r & t^{*}
\end{array}\right)=\left(\begin{array}{cc}
1 / t^{\prime *} & r^{\prime} / t^{\prime} \\
r^{\prime *} / t^{\prime *} & 1 / t^{\prime}
\end{array}\right)
$$

The second equality comes from an alternative form of the $S$ matrix expressed in terms of $r^{\prime}$ and $t^{\prime}$, by considering an em wave incident from the right. Equating the two alternative forms of the $S$ matrix [Eq. (A9)] element by element, we obtain

$$
\begin{gathered}
1-|r|^{2}=t t^{\prime *}, \\
\frac{-r^{*}}{t^{\prime *}}=\frac{r^{\prime}}{t^{\prime}} .
\end{gathered}
$$

\section{TE mode: Conservation of energy}

Consider now the conservation of energy current. In a lossless and source-free medium, the conservation of energy implies that

$$
\int \operatorname{Re} \vec{S} \cdot \overrightarrow{d A}=0
$$

where $\vec{S}=\frac{1}{2}\left(\vec{E} \times \vec{H}^{*}\right)$ is the Poynting vector and the integral has been taken on a closed surface.

The Poynting vector can be easily computed for the asymptotic forms of the em fields. For example, for the $\vec{E}$ field in Eq. (A3), we have

$$
\begin{aligned}
\vec{S}= & \frac{1}{2 \mu \omega}\left\{i k_{x}\left[\left|A_{L}\right|^{2}-\left|B_{L}\right|^{2}-\left(A_{L} B_{L}^{*} e^{2 i k_{x} x}-\text { c.c. }\right)\right]\right. \\
& \left.+j k_{y}\left[\left|A_{L}\right|^{2}+\left|B_{L}\right|^{2}+\left(A_{L} B_{L}^{*} e^{2 i k_{x} x}+\text { c.c. }\right)\right]\right\} .
\end{aligned}
$$

This form is valid everywhere and not just in the asymptotic region, provided that we make the segmentation into regions of constant $\epsilon$ and $\mu$. Inspection of Eq. (A12) shows that

$$
\begin{aligned}
& \frac{\partial\left(\operatorname{Re} S_{x}\right)}{\partial x}=0, \\
& \frac{\partial\left(\operatorname{Re} S_{y}\right)}{\partial y}=0 .
\end{aligned}
$$

Applying the conservation relation (A11) to a rectangular box, with faces normal to the Cartesian coordinates, and using the relation (A13), we find that

$$
\hat{i} \cdot \operatorname{Re} \vec{S}=\text { const }
$$

in all space. This is easily shown to be true for the TM mode as well.

The conservation relation (A14) applied to the asymptotic solution (A3) and the resulting expression (A12) for $\vec{S}$ leads to the condition that

$$
\frac{k_{x}}{\mu_{1}}\left(\left|A_{L}\right|^{2}-\left|B_{L}\right|^{2}\right)=\frac{\bar{k}_{x}}{\mu_{2}}\left(\left|A_{R}\right|^{2}-\left|B_{R}\right|^{2}\right)
$$

Substituting the right coefficients $A_{R}, B_{R}$ in terms of $A_{L}, B_{L}$, and the $S$ matrix, we find

$$
\frac{k_{x}}{\mu_{1}}=\frac{\bar{k}_{x}}{\mu_{2}}\left(\left|s_{11}\right|^{2}-\left|s_{12}\right|^{2}\right),
$$

which in turn leads to the relation

$$
|r|^{2}+\frac{\bar{k}_{x} \mu_{1}}{k_{x} \mu_{2}}|t|^{2}=1
$$

Using the analogous equation for $r^{\prime}$ and $t^{\prime}$, plus the fact that $|r|=\left|r^{\prime}\right|$ [which follows from Eq. (A10)], we find that

$$
\left|t^{\prime}\right|=\left(\frac{\bar{k}_{x} \mu_{1}}{k_{x} \mu_{2}}\right)|t|
$$

\section{TE mode: Relationship between the left and the right transport coefficients: $r, t$ and $r^{\prime}, t^{\prime}$}

Consider em waves of equal amplitude incident from both sides of the dielectric. Applying the energy conservation (A14), we find that

$$
\frac{k_{x}}{\mu_{1}}\left(1-\left|r+t^{\prime}\right|^{2}\right)=\frac{\bar{k}_{x}}{\mu_{2}}\left(\left|r^{\prime}+t\right|^{2}-1\right),
$$

which leads to the condition

$$
\operatorname{Re}\left(\frac{k_{x} \mu_{2}}{\bar{k}_{x} \mu_{1}} r t^{\prime *}+r^{\prime} t^{*}\right)=0 .
$$

The most general form of the transport coefficients satisfying all relations between them is that

$$
\begin{gathered}
r=|r| e^{i \delta}, \\
t=|t| e^{i \eta}, \\
r^{\prime}=-|r| e^{i(2 \eta-\delta)}, \\
t^{\prime}=\left(\frac{\bar{k}_{x} \mu_{1}}{k_{x} \mu_{2}}\right)|t| e^{i \eta} .
\end{gathered}
$$

\section{TE mode: Symmetric dielectric}

The above equations are valid for both a symmetric and nonsymmetric dielectric. Consider now the symmetric dielectric, $\epsilon(-x)=\epsilon(x)$ and $\mu(-x)=\mu(x)$, on which an em wave is incident from both the left and right with equal amplitudes. From symmetry, the energy current is zero everywhere. Computing the current in the asymptotic region $x \rightarrow$ $-\infty$, we obtain

$$
\hat{i} \cdot \operatorname{Re} \vec{S}=\frac{k_{x}}{2 \mu_{1} \omega}\left(1-\left|r+t^{\prime}\right|^{2}\right)=0,
$$

which leads to the condition that

$$
\operatorname{Re}\left(r t^{*}\right)=0
$$


i.e., $r t^{*}=$ purely imaginary, where we have used the relations $t=t^{\prime}$ [Eq. (A21)] and $|r|^{2}+|t|^{2}=1$ [Eq. (A17)]. Equation (A23) together with Eq. (A21) leads to the result

$$
\begin{gathered}
r=r^{\prime}= \pm i|r| e^{i \eta}, \\
t=t^{\prime}=|t| e^{i \eta},
\end{gathered}
$$

for the symmetric case. In addition, from Eq. (A17), we also have

$$
|r|^{2}+|t|^{2}=1
$$

Note that if the dielectric structure is symmetric but only about the point $x=x_{0}$ [i.e., $\epsilon\left(x_{0}-x\right)=\epsilon\left(x-x_{0}\right)$ and similarly for $\mu]$, then the em waves $e^{i k x}$ incident from the left and $e^{-i k x}$ incident from the right do not produce a zero current, because the two incident waves approach the structure with different phases. Therefore, Eqs. (A24) and (A25) are not valid in that case.

\section{TM mode}

For the TM mode, we choose to work with the $\vec{B}$ field. The Maxwell's wave equation is

$$
\nabla^{2} \vec{B}=-\frac{\omega^{2} n^{2}}{c^{2}} \vec{B}
$$

With $\vec{B}$ along $\hat{z}$, this equation is exactly the same as the corresponding Eq. (A1) for the $\vec{E}$ field with $\vec{E}$ along $\hat{z}$. Therefore all asymptotic properties of the differential equation are identical. Therefore, all relations between the transport coefficients $r, t$ and $r^{\prime}, t^{\prime}$ derived for the TE case also hold true for the TM case. Note, however, that since $\vec{E}$ and $\vec{B}$ satisfy different boundary conditions at the interface between two dielectric media, the numerical values of the reflection and transmission coefficients and the $S$ matrix will be different in general.
${ }^{1}$ E. Yablonovitch and T. J. Gmitter, Phys. Rev. Lett. 63, 1950 (1989).

${ }^{2}$ Z. Zhang and S. Satpathy, Phys. Rev. Lett. 65, 2650 (1990); K. M. Leung and Y. F. Liu, ibid. 65, 2646 (1990); K. M. Ho, C. T. Chan, and C. M. Soukoulis, ibid., 65, 3152 (1990).

${ }^{3}$ H. L. Skriver, The LMTO Method (Springer, New York, 1983); O. K. Andersen, Phys. Rev. B 12, 3060 (1975).

${ }^{4}$ M. Born and E. Wolf, Principles of Optics, 7th ed. (Cambridge University, Cambridge, England, 1999), Sect. 1.5.

${ }^{5}$ S. Mishra and S. Satpathy, Am. J. Phys. 69, 512 (2001).

${ }^{6}$ N. W. Ashcroft and N. D. Mermin, Solid State Physics (Saunders, Philadelphia, 1976), p. 148.
${ }^{7}$ P. Yeh, A. Yariv, and C. S. Hong, J. Opt. Soc. Am. 67, 423 (1977); P. Yeh, Optical Waves in Layered Media (Wiley, New York, 1988), Chap. 6.

${ }^{8}$ See, for example, G. Grosso and G. P. Parravicini, Solid State Physics (Academic, London, 2000).

${ }^{9}$ F. L. Pedrotti and L. S. Pedrotti, Introduction to Optics, 2nd ed. (Prentice-Hall, Englewood Cliffe, NJ, 1993), p. 395.

${ }^{10}$ N. Liu, Phys. Rev. B 55, 4097 (1997).

${ }^{11}$ J. B. Pendry, J. Phys.: Condens. Matter 8, 1085 (1996).

${ }^{12}$ J. D. Joannapoulous, R. D. Meade, and J. N. Winn, Photonic Crystrals (Princeton University, Princeton, NJ, 1995). 